\title{
Multi-Objective Optimization for an RFID-Enabled Automated Warehousing System
}

\author{
Ahmed Mohammed, Saleh Alyahya \\ School of Engineering, University of Portsmouth \\ Portsmouth, UK
}

\author{
Qian Wang \\ School of Engineering, University of Portsmouth \\ Portsmouth, UK
}

\begin{abstract}
Over the last decade, it has been seen a rapid pace in research and development of RFID-based automatic storage and retrieval systems (AS/RSs) due to the usage of increasingly centralized distribution centers to warehouse merchandising products, which are sold using the online method. This paper presents a study of an RFID-enabled warehousing system as part of the research work for future generation warehouses design. To this aim, a multi-objective optimization model was developed and used for obtaining trade-off decisions by measuring four conflicted objectives: minimization of the total cost, maximization of capacity utilization, maximization of service level and minimization of travel distance in the warehouse. The study also supports design decisions in determining an optimum number of storage racks and collection points that need be established for the warehouse. To reveal the alternative Pareto-optimal solutions using the developed model, an integrated fuzzy solution approach was proposed. Subsequently, a decision making algorithm was used to select the best Pareto-optimal solution. A case-study was conducted to demonstrate the applicability of the developed model.
\end{abstract}

Keywords-Warehouses; RFID; optimization; multi-objective

\section{INTRODUCTION}

Warehouses are one of main components which consist of an entire supply chain. Usually, warehouses receive and store merchandising products from suppliers before being transported to retailers or customers. In the last decade it has seen a growing need towards a trend in application and implementation of automated warehouses aiming to improve efficiency and capacity utilization, and reduce delivery time from warehouses to customers.

There are a limited number of research publications in optimization of automated warehouse design in a combination of considerations including travel time, costs, and capacity utilization. Wang [1] presented a study of an RFID-based automated warehousing mechanism in order to address the tighter inventory control, shorter response time and greater variety of SKUs (stock keeping units), which are the most important challenges for designing future generation warehouses. Ma [2] formulated an automated warehouse as a constrained multi-objective model aimed at minimizing the scheduling quality effect and the travel distance. Huang [3] proposed a nonlinear mixed integer program under probabilistic constraints for site selection and space determination of warehouses by minimizing the total inbound and outbound transportation cost and the total warehouse operation cost in a two-stage network. Lerher [4] investigated the design in optimization of an automated storage and retrieval system aiming to minimize the initial investment and annual operating cost of the system. A genetic algorithm was used for the optimization process of decision variables. Lerher [5] proposed a mono-objective optimization approach for seeking the cost-effective design of an automated warehouse. Ashayeri [6] developed a design model of an automated storage and retrieval system incorporating the main influential parameters to minimize costs in investment and operation. Karasawa [7] developed a nonlinear mixed integer model aimed at minimizing the system cost for an automated warehouse system.

In brief, a literature review in this area indicates there are limited studies in previous publications in optimizing the design of RFID-based automated warehousing system using the multi-objective method. This paper attempts to address this issue using the multi-objective method as an aid for optimizing the design of a proposed RFID-enabled automated warehousing system. To this aim, a multiobjective optimization model was developed to maximize the warehouse capacity utilization and service level, and minimize travel distance of products from a storage rack to a collection point and minimize the total cost required for implementing such a warehousing system. The paper also includes an investigation in configuration of the proposed warehousing system focusing on the optimal number of racks and collection points that need be established.

\section{PROBLEM DEFINITION AND MODEL FORMULATION}

Figure 1 illustrates the structure of the concerned RFIDenabled AS/RR [1]. The module comprises of two types of powered conveyors aligned next to one another; these are input conveyors (storage racks) and output conveyors. The entire operation of each conveyor system is controlled by a programmable logic controller that communicates with mounted sensors via a local area network. Within the RFIDinventory management system, a chosen stock keeping unit 
(SKU) can be released by the system based on a number of assignment policies or rules. These rules include for example the rule of being nearest to a collection point and/or a modular arm which is free or adjacent to the chosen SKU.

One of the main issues to be addressed in designing the proposed RFID-enabled automated warehouse include allocating the optimum number of racks and collection points with respect to four objective functions: (1) minimization of total cost of implementing the warehouse,

(2) maximization of capacity utilization of the warehouse,

(3) maximization of service level in terms of satisfying all demands of dispatching products from the warehouse and (4) minimization of travel distance of products from racks to collection points.

The following sets, parameters and decision variables were used in the formulation of the model:

Sets:

$\begin{array}{ll}I & \text { set of racks } i \in I \\ J & \text { set of collection points } j \in J \\ K & \text { set of departure points } k \in K\end{array}$

Given parameters:

$C_{i}^{r} \quad$ fixed cost required for establishing an RFID-enabled rack $i$

$C_{i}^{c} \quad$ fixed cost required for establishing a collection point $j$

$C_{i}^{t} \quad$ unit RFID tag cost per item at rack $i$

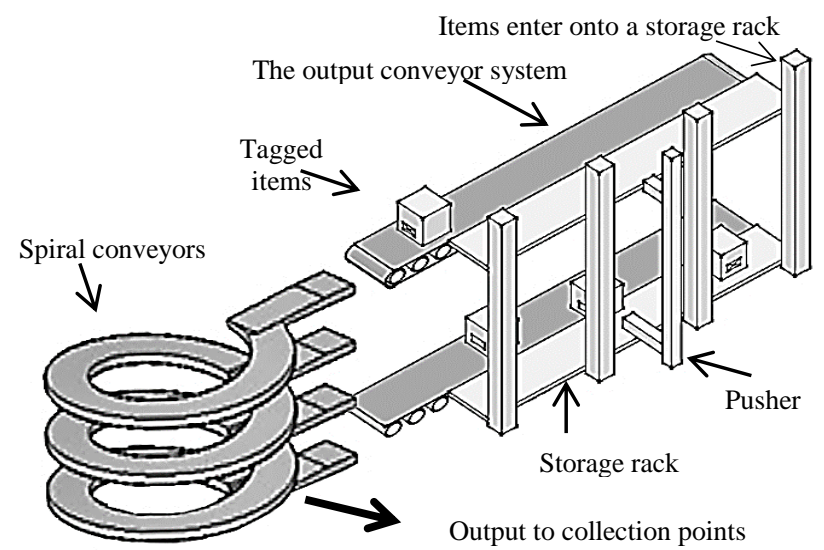

Figure 1. Structure of the RFID-enabled AS/RR [1].

$C_{j k}^{\mathrm{T}} \quad$ unit transportation (T) cost per meter from collection point $j$ to departure point $k$

$C_{j}^{l} \quad$ unit labor cost per hour at collection point $j$

$R_{j}^{l} \quad$ working rate (items) per laborer $(l)$ at collection point $j$

$d_{j k} \quad$ travel distance (meter) per item from collection point $j$ to departure point $k$

$W \quad$ transportation capacity (units) per forklift

$\mathrm{S}_{\mathrm{i}} \quad$ maximum supply capacity (units) of rack $i$
$\mathrm{S}_{\mathrm{j}} \quad$ maximum supply capacity (units) at collection point j

$\mathrm{D}_{\mathrm{j}} \quad$ demand (in units) at collection point $j$

$t_{i j}^{d} \quad$ average travel distance (meter) per item from rack $i$ to collection point $j$

Decision variables

$q_{\mathrm{ij}} \quad$ quantity of units travelling from rack $i$ to collection point $j$

$q_{j k} \quad$ quantity of units dispatched from collection point $j$ to departure point $k$

$x_{j} \quad$ required number of laborers at collection point $j$

$\mathrm{y}_{\mathrm{i}}\left\{\begin{array}{l}1: \text { if rack } i \text { is required } \\ 0: \text { otherwise }\end{array}\right.$

$\mathrm{y}_{\mathrm{j}}\left\{\begin{array}{l}1: \text { if collection point } j \text { is required } \\ 0: \text { otherwise }\end{array}\right.$

The four objectives, which include a minimization of total cost, a maximization of capacity utilization, maximization of service level and minimization of travel distance, are formulated as follows:

Objective function (1)

In this case, the total cost of establishing the RFIDenabled automated warehouse includes costs of establishing RFID-enabled racks, collection points, RFID tag, transportation of products and labors. Thus, minimization of the total cost can be expressed below:

$$
\begin{aligned}
& \operatorname{Min} F_{1}=\sum_{i \in I} C_{i}^{r} y_{i}+\sum_{j \in J} C_{j}^{c} y_{j}+\sum_{i \in I} \sum_{j \in j} C_{i}^{t} q_{i j} \\
& +\sum_{j \in J} \sum_{k \in K} C_{i j}^{T}\left\lceil q_{j k} / W_{f}\right\rceil d_{j k}+\sum_{i \in I} C_{i}^{l} x_{j}
\end{aligned}
$$

Objective function (2)

Maximization of capacity (C) utilization is expressed as follows:

$$
\operatorname{Max} F_{2}=\left(\sum_{i \in I} \frac{\left[\left(C_{\text {actual }}\right)-\left(C_{\text {used }}\right)\right]^{2}}{\sum i}\right)^{\frac{1}{2}}
$$

Where $C_{\text {actual }}=\sum_{j \in J} \frac{q_{i j}}{S_{i}}$ and $C_{\text {used }}=\frac{\sum_{i \in I} \sum_{j \in J} q_{i j}}{\sum_{i \in I} S_{i}}$.

Objective function (3)

To ensure the satisfaction of all demands of products delivered by the warehouse, this can be achieved by maximization of service level, which is given by:

$$
\operatorname{Max} F_{3}=\sum_{i \in I} \sum_{j \in J} \frac{q_{i j}}{D_{i}}
$$

Objective function (4) 
The travel distance of an in-store item from its location of a storage rack to a collection point can be minimized as follows:

$$
\operatorname{Min} F_{4}=\sum_{i \in I} \sum_{j \in J} t_{i j}^{d} q_{i j}
$$

There are a number of constraints which are included in the optimization. The constraints are given as follows:

$$
\begin{array}{cc}
\sum_{i \in I} q_{i j} \leq S_{i} y_{i} & \forall j \in J \\
\sum_{j \in J} q_{j k} \leq S_{j} y_{j} & \forall k \in K \\
\sum_{i \in I} q_{i j} \geq D_{j} & \forall j \in J \\
D_{j} \geq \sum_{k \in K} q_{j k} & \forall j \in J \\
\sum_{j \in J} q_{i j} \leq x_{j} \mathrm{R}_{j}^{1} & \forall \mathrm{i} \in I \\
q_{i j}, q_{j k} \geq 0, \quad \forall i, j, k ; \\
y_{i}, y_{j} \in\{0,1\}, \forall i, j ;
\end{array}
$$

Equations 5 and 6 refer to the flow balance of a product travelling from a storage rack to a collection point and from a collection points to a departure point. Equations 7 and 8 refer to demands to be satisfied. Equation (9) determines the required number of labors at a collection point. Equations (10) and (11) limit the decision variables to binary and nonnegative.

\section{OPTIMIZATION METHODOLOGY}

Several approaches were reported in the literature to solve the multi-objective problem. In this work, a fuzzy solution approach was proposed to transform the multiobjective model into a single-objective model which is formulated by considering each objective individually by minimizing the scalarized difference between value of each objective and its optimal value. Undesired deviations were used for being subtracted from the single objective function to obtain more accurate objective values.

\section{A. Solution procedures}

To solve the developed multi-objective model, the solution procedures are expressed as follows:

1) Convert the developed model into an equivalent crisp model using Jiménez method [8]. Accordingly, the equivalent crisp model can be formulated as follows.

$$
\begin{gathered}
\text { Min } F_{1}=\sum_{i \in I} \sum_{p=1}^{4} \frac{C_{i p}^{r}}{4} y_{i}+\sum_{j \in J} \sum_{p=1}^{4} \frac{C_{j p}^{c}}{4} y_{j}+\sum_{i \in I} \sum_{j \in j} \sum_{p=1}^{4} \frac{C_{i p}^{t}}{4} q_{i j} \\
+\sum_{j \in J} \sum_{k \in K} \sum_{p=1}^{4} \frac{C_{i j p}^{T}}{4}\left\lceil q_{j k} / W_{f}\right] d_{j k}+\sum_{i \in I} \sum_{p=1}^{4} \frac{C_{i p}^{l}}{4} x_{j} \\
\operatorname{Max}_{2}=\left(\sum_{i \in I} \frac{\left\lceil\left(C_{\text {actual }}\right)-\left(C_{\text {used }}\right)\right]^{2}}{\sum i}\right)^{\frac{1}{2}}
\end{gathered}
$$

$$
\begin{aligned}
\operatorname{Max}_{3} & =\sum_{i \in I} \sum_{j \in J} \sum_{p=1}^{4} 4 \frac{q_{i j}}{D_{i p}} \\
\operatorname{Min} F_{4} & =\sum_{i \in I} \sum_{j \in J} t_{i j}^{d} q_{i j}
\end{aligned}
$$

Subject to:

$$
\begin{gathered}
\sum_{i \in I} q_{i j} \leq S_{i} y_{i} \quad \forall j \in J \\
\sum_{j \in J} q_{j k} \leq S_{j} y_{j} \quad \forall k \in K \\
\sum_{i \in I} q_{i j} \geq \frac{\lambda}{2} \frac{D_{j 1}+D_{j 2}}{2}+\left(1-\frac{\lambda}{2}\right) \frac{D_{j 3}+D_{j 4}}{2} \quad \forall j \in J \\
\frac{\lambda}{2} \cdot \frac{D_{j 1}+D_{j 2}}{2}+\left(1-\frac{\lambda}{2}\right) \frac{D_{j 3}+D_{j 4}}{2} \geq \sum_{k \in K} q_{j k} \quad \forall j \in J \\
\sum_{j \in J} q_{i j} \leq x_{j} \frac{\lambda}{2} \cdot \frac{x_{j 1}+x_{j 2}}{2}+\left(1-\frac{\lambda}{2}\right) \frac{x_{j 3}+x_{j 4}}{2} \mathrm{R}_{j}^{1} \quad \forall \mathrm{i} \in I \\
q_{i j}, q_{j k} \geq 0, \forall i, j, k ; \\
y_{i}, y_{j} \in\{0,1\}, \forall i, j ;
\end{gathered}
$$

2) Find the upper and lower bound (U, L) solution for each objective function. This can be obtained as follows for Upper bound solutions:

$\operatorname{Max} F_{1}\left(U_{1}\right)=\sum_{i \in I} C_{i}^{r} y_{i}+\sum_{j \in J} C_{j}^{c} y_{j}+\sum_{i \in I} \sum_{j \in j} C_{i}^{t} q_{i j}$

$+\sum_{j \in J} \sum_{k \in K} C_{i j}^{T}\left\lceil q_{j k} / W_{f}\right\rceil d_{j k}+\sum_{i \in I} C_{i}^{l} x_{j}$

$$
\begin{gathered}
\operatorname{Max} F_{2}\left(U_{2}\right)=\left(\sum_{i \in I} \frac{\left\lceil\left(C_{\text {actual }}\right)-\left(C_{\text {used }}\right)\right\rceil^{2}}{\sum i}\right)^{\frac{1}{2}} \\
\operatorname{Max}_{3}\left(U_{3}\right)=\sum_{i \in I} \sum_{j \in J} \frac{q_{i j}}{D_{i}} \\
\operatorname{Max}_{4}\left(U_{4}\right)=\sum_{i \in I} \sum_{j \in J} t_{i j}^{d} q_{i j}
\end{gathered}
$$

Lower bound solutions:

$$
\begin{gathered}
\operatorname{Min} F_{1}\left(U_{1}\right)=\sum_{i \in I} C_{i}^{r} y_{i}+\sum_{j \in J} C_{j}^{c} y_{j}+\sum_{i \in I} \sum_{j \in j} C_{i}^{t} q_{i j} \\
+\sum_{j \in J} \sum_{k \in K} C_{i j}^{T}\left\lceil q_{j k} / W_{f}\right\rceil d_{j k}+\sum_{i \in I} C_{i}^{l} x_{j} \\
\operatorname{Min} F_{2}\left(U_{2}\right)=\left(\sum_{i \in I} \frac{\left\lceil\left(C_{\text {actual }}\right)-\left(C_{\text {used }}\right)\right\rceil^{2}}{\sum^{\frac{1}{2}} i}\right)^{\frac{1}{2}} \\
\operatorname{Min} F_{3}\left(U_{3}\right)=\sum_{i \in I} \sum_{j \in J} \frac{q_{i j}}{D_{i}} \\
\operatorname{Min} F_{4}\left(U_{4}\right)=\sum_{i \in I} \sum_{j \in J} t_{i j}^{d} q_{i j}
\end{gathered}
$$

3) Find the respective satisfaction degree $\mu$ (xi) for each objective function as follows: 


$$
\mu_{i}(F i(x))= \begin{cases}1 & \text { if } F_{i}(x) \geq U_{i} \\ \frac{F_{i}(x)-L_{i}}{U_{i}-L_{i}} & \text { if } L_{i} \leq F_{i}(x) \leq U_{i}, i=1,2,3,4 \\ 0 & \text { if } F_{i}(x) \leq L_{i}\end{cases}
$$

4) Transform the crisp model obtained from step (1) to a single objective function using the developed solution method. The developed solution method function $(\mathrm{F})$ is formulated as follows:

$$
\operatorname{Min} F=\left(\sum_{n=1}^{4} \sum_{f=1}^{4} \vartheta_{n} \mu_{f}(x)\right)-F_{d}, \sum_{n=1}^{4} \vartheta_{n}=1
$$

Set $\vartheta_{n}^{*}=\frac{\vartheta_{n} F_{n}^{\bullet}}{F_{n}^{\bullet}-F_{n}}$, then

$$
\begin{aligned}
& F_{d}=\vartheta_{1}^{*} F_{1}+\vartheta_{2}^{*} F_{2}+\vartheta_{3}^{*} F_{3}+\vartheta_{4}^{*} F_{4} \\
& =\frac{\vartheta_{1} F_{1}^{\bullet}}{F_{1}^{*}-F_{1}} F_{1}+\frac{\vartheta_{2} F_{2}^{\bullet}}{F_{2}^{\bullet}-F_{2}} F_{Z}+\frac{\vartheta_{3} F_{3}^{\bullet}}{F_{3}^{\bullet}-F_{3}} F_{3}+\frac{\vartheta_{4} F_{4}^{\bullet}}{F_{4}^{*}-F_{4}} F_{4}
\end{aligned}
$$

Based on the aforementioned procedures, the developed method's objective function can be written as follows.

$\operatorname{Min} F=\left(\vartheta_{1} \mu_{1}-\vartheta_{2} \mu_{2}-\vartheta_{3} \mu_{3}-\vartheta_{4} \mu_{4}\right)$

$-\left(\frac{\vartheta_{1} F_{1}^{\cdot}}{F_{1}^{*}-F_{1}} F_{1}+\frac{\vartheta_{2} F_{2}^{\cdot}}{F_{2}^{*}-F_{2}} F_{2}+\frac{\vartheta_{3} F_{3}^{\cdot}}{F_{3}^{*}-F_{3}} F_{3}+\frac{\vartheta_{4} F_{4}^{\cdot}}{F_{4}^{\cdot}-F_{4}} F_{4}\right)$

Subject to equations (5)-(11).

5) Vary the weight combination set $(\vartheta)$ consistently for the four objectives to reveal Pareto-optimal solutions. Usually, the weight combination set is allocated by decision makers based on the importance of each objective.

6) Select the best Pareto-optimal solution using the proposed decision making algorithm.

\section{B. The decision making algorithm}

The next step after revealing the Pareto solutions is to determine the best trade-off solution. In this work, Technique for order preference by similarity to ideal solution (TOPSIS) was employed for revealing the best trade-off solution. This approach selects not merely the closest solution to the ideal solution, but also the farthest from the negative ideal solution [9].

\section{Assume}

PR- $\left\{\begin{array}{l}\mathrm{PR}_{\mathrm{op}} \mid \mathrm{o}=1,2, \ldots, \mathrm{X} \text { (number of pareto solutions); } \mathrm{p}=1,2, \ldots, \\ \mathrm{y} \text { (number of objectives) }\end{array}\right\}$

refers the $x^{*} y$ decision matrix, where $P R$ is the performance rating of alternative Pareto solutions with respect to objective function values. Thus, the normalized selection formula is presented as follows:

$$
N P R=\frac{P R_{o p}}{\sum_{p=1}^{o} P R_{a p}}
$$

The amount of decision information can be measured by the entropy value as:

$$
E_{p}=\frac{-1}{\ln \mathrm{x}} \sum_{o=1}^{x} P R_{o p} \ln \left(P R_{o p}\right)
$$

The degree of divergence $\left(D_{p}\right)$ of the average intrinsic information contained for $p=1,2,3,4$ can be calculated as:

$$
D_{p}=1-E_{p}
$$

The weight for each objective function value is given by:

$$
w_{p}=\frac{D_{p}}{\sum_{k=1}^{y} D_{k}}
$$
by:

Thus, the objective weighted normalized value is given

$$
v_{o p}=w_{o} P R_{o p}
$$

The positive ideal solution AT and the negative ideal solution At are taken to generate an overall performance matrix for each Pareto solution. These values can be expressed as:

$$
\begin{aligned}
& A T^{+}=\left(\max \left(v_{o 1}\right) \max \left(v_{o 2}\right) \max \left(v_{o y}\right)\right)=\left(v_{1}^{+}, v_{2}^{+}, \ldots, v_{y}^{+}\right) \\
& A T^{-}=\left(\min \left(v_{o 1}\right) \min \left(v_{o 2}\right) \quad \min \left(v_{o y}\right)\right)=\left(v_{1}^{-}, v_{2}^{-}, \ldots, v_{y}^{-}\right)
\end{aligned}
$$

Distance between alternative solutions can be measured by the n-dimensional Euclidean distance. The separation of each alternative from the ideal solution is given as

$$
D_{p}^{+/-}=\sqrt{\left\{\sum_{o=1}^{y}\left(v_{p o}-v_{o}^{+/-}\right)^{2}\right\}}, \quad p=1,2, \ldots, x
$$

The relative closeness to the ideal solution of alternative solutions with respect to objective function values is expressed as follows:

$$
r c_{p}=\frac{D_{p}^{-}}{D_{p}^{+}+D_{p}^{-}}, \quad p=1,2, \ldots, x
$$

Where $D_{p}^{-} \geq 0$ and $D_{p}^{+} \geq 0$, then, clearly, $r c_{p} \in[1,0]$. Select the trade-off solution with maximum $r c_{p}$ or list the obtained solution in descending order based on $r c_{p}$.

\section{APPLICATION AND EVALUATION}

In this section, a case study was used to investigate the applicability of the developed optimization model and the performance of the proposed optimization methodology. Table I shows the parameters used for application. The solver for the developed multi-objective model was LINGO $^{11}$.

\section{A. Results and discussions}

This section presents the computational results of the developed multi-objective model using the proposed integrated fuzzy solution approach for the problem previously defined. The solution steps of the developed model are described as follows:

1) Obtain the upper and lower value for each objective function by solving them individually. The results are $\left(\left\{U_{F}\right.\right.$, $\left.\left.L_{F}\right\}\right)=(\{504,1,230\},\{0.66,0.94\},\{0.85,0.99\},\{400$,

TABLE I. APPLICATION DATA RANGES

$\begin{array}{rlrl}I & =12 & S_{i}=25 \mathrm{~K}-35 \mathrm{~K} \\ J & =15 & S_{j}=20-29 \mathrm{~K} \\ K & =2 & & \mathrm{~d}_{j k}=20-45 \\ \mathrm{C}_{j}^{l} & =6.5-9(\mathrm{GBP}) & & t_{\mathrm{ij}}^{d}=40-75 \\ \mathrm{C}_{\mathrm{i}}^{r} & =60-90(\mathrm{KGBP}) & \end{array}$




\begin{tabular}{ll}
$\mathrm{C}_{i}^{t}=0.25(\mathrm{GBP})$ & $\mathrm{D}_{\mathrm{j}}=150-360 \mathrm{~K}$ \\
$\mathrm{C}_{j k}^{T}=0.4(\mathrm{GBP})$ & $\mathrm{d}_{j k}=20-45$ \\
$R_{j}^{l}=100$ & $C_{j}^{c}=15-18(\mathrm{KGBP})$ \\
$W=48$ & \\
\hline
\end{tabular}

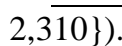

2) Find the respective satisfaction degree $\mu$ (xi) for each objective function. The satisfaction degrees are reported in Table II.

3) Solve the crisp model as a single objective model using the developed solution method by an assignment of different combination of weight values with respect to the feasibility of each weight pair that is denoted by $\lambda$.

4) Select the best solution using TOPSIS, the determined score values of Pareto-optimal solutions are reported in Table III.

Table IV shows the obtained set of Pareto-optimal solutions for the four objective functions. It also shows the number of racks and collection points that should be established. For instance, solution 1, which is obtained by an assignment of $\vartheta_{1}=1, \vartheta_{2}=0, \vartheta_{3}=0$ and $\vartheta_{4}=0$, has a minimum total cost of $504 \mathrm{KGBP}$, a maximum capacity utilization of $66 \%$, a maximum service level of $85 \%$ and a minimum travel distance of $400 \mathrm{~km}$ of all products. This solution offers six racks and nine collection points. Figure 2 illustrates the further comparison among the solutions in response to the four objective functions. It can be observed in Figure 2 that the Pareto optimal method cannot produce a better solution in one objection function without worsening its performance in the other objective functions.

After obtaining a set of Pareto-optimal solutions, decision makers may determine a solution depending on their preferences or using a decision making algorithm. In this work, TOPSIS was employed to select the best solution. As shown in Table III, solution 4 is the best solution as its score is the highest (0.279) with the total cost of $759 \mathrm{~K} \mathrm{GBP}$, $77 \%$ capacity utilization, $95.5 \%$ service level and travel distance $1021 \mathrm{~km}$.

TABLE II. SATISFACTION DEGREES FOR OBJECTIVE FUNCTIONS

\begin{tabular}{lllllllll}
\hline$\mu\left(x_{1}\right)$ & 0.95 & 0.93 & 0.85 & 0.81 & 0.7 & 0.623 & 0.6 & 0.55 \\
$\mu\left(x_{2}\right)$ & 0.7 & 0.78 & 0.83 & 0.88 & 0.92 & 0.97 & 0.98 & 0.99 \\
$\mu\left(x_{3}\right)$ & 0.73 & 0.79 & 0.85 & 0.89 & 0.919 & 0.96 & 0.98 & 0.99 \\
$\mu\left(x_{4}\right)$ & 0.97 & 0.96 & 0.93 & 0.90 & 0.85 & 0.84 & 0.81 & 0.76 \\
\hline
\end{tabular}

TABLE III. SCORE VALUES OF PARETO-OPTIMAL SOLUTION USING TOPSIS

\begin{tabular}{lllllllll}
\hline$\#$ & 1 & 2 & 3 & 4 & 5 & 6 & 7 & 8 \\
Score & 0.245 & 0.234 & 0.266 & 0.279 & 0.254 & 0.245 & 0.273 & 0.243 \\
\hline
\end{tabular}

Also, it requires an establishment of nine racks to supply products to eleven collection points.

\section{CONCLUSIONS}

In this work, a proposed RFID-enabled automated warehousing system was studied using the multi-objective optimization approach. The problem was involved in obtaining trade-offs between the negative impact (the extra costs) and the positive impact (maximization of the warehouse capacity utilization and service level, and minimization of travel distance of products from racks to collection points) of the proposed RFID-enabled automated warehousing system. The study also includes an approach to determine the optimal number of racks and collection points that should be established when designing the proposed warehouse. At the first stage, an integrated fuzzy solution approach was used to obtain Pareto-optima solutions. At the second stage, TOPSIS was employed to reveal the best Pareto solution. By implementation of the developed model using a case study it proves a reasonable applicability. The obtained solutions are close enough to ideal solutions since undesired deviations are proposed to be subtracted from the objective functions. This leads to a clear insight of a compromise solution between conflicting objectives for decision makers.

\section{REFERENCES}

[1] Q.Wang, R.McIntosh, M.Brain, "A new-generation automated warehousing capability", International Journal of Computer Integrated Manufacturing, Vol. 23, No. 6, pp. 565-573, 2010.

[2] H. Ma, S. Su, D. Simon, and M. Fei, "Ensemble multi-objective biogeography-based optimization with application to automated warehouse scheduling," Engineering Applications of Artificial Intelligence, vol. 44, 2015, 79-90,

[3] S. Huang, Q. Wang, R. Batta, and R. Nagi, "An integrated model for site selection and space determination of warehouses," Computers \& Operations Research, vol. 62, 2015, pp. 169-176, doi: 10.1016/j.cor.2014.10.015.

[4] T. Lerher, I. Šraml, M. Borovinšek, and I. Potrč, "Multi-objective optimization of automated storage and retrieval systems," Annals of faculty of mechanical engineering-International journal of Engineering, 2013, 187-194.

[5] T. Lerher, I. Potrc, and M. Sraml, "Designing automated warehouses by minimising investment cost using genetic algorithms," Progress in material handling research: 2010. Charlotte: The Material Handling Industry of America, cop. 2013, 237-253.

[6] J. Ashayeri, and L.F. Gelders, "A microcomputer-based optimization model for the design of automated warehouses," International Journal of Production Research, vol. 23, 1985, pp. 825- 839, doi: $10.1080 / 00207548508904750$.

[7] Y. Karasawa, H. Nakayama, and S. Dohi, "Trade-off analysis for optimal design of automated warehouses," International Journal of System Science, vol. 11, 1980, pp. 567-576, doi: 10.1080/00207728008967037.

[8] M. Jiménez López, M.V. Rodríguez Uría, M.A. Parra, and A. Bilbao, "Solving a possibilistic linear program through compromise programming," Math ware \& Soft Computing, vol. 7, 2000, pp. 175184.

[9] S. Ramesh, S. Kannan, and S. Baskar, "Application of modified NSGA-II algorithmto multi-objective reactive power planning," Appl. Soft Comput, vol. 12, 2013, pp. 741-753, doi: 10.1016/j.asoc.2011.09.015. 
TABLE IV. COMPUTATIONAL RESULTS

\begin{tabular}{cccccccc}
\hline \multicolumn{6}{c}{ 350 non-zero elements, 59 constraints, 111 total variables, 59 integer variables } \\
\hline $\begin{array}{c}\text { Feasibility } \\
\text { level }\end{array}$ & $\#$ & $\begin{array}{c}\text { Min }\left(\boldsymbol{F}_{2}\right) \\
(\boldsymbol{K G B P})\end{array}$ & $\begin{array}{c}\text { Max }\left(\boldsymbol{F}_{\mathbf{2}}\right) \\
(\boldsymbol{\%})\end{array}$ & $\begin{array}{c}\boldsymbol{M a x}\left(\boldsymbol{F}_{3}\right) \\
(\%)\end{array}$ & $\begin{array}{c}\text { Min }\left(\boldsymbol{F}_{4}\right) \\
(\text { Km })\end{array}$ & Open racks & $\begin{array}{c}\text { Open collection } \\
\text { points }\end{array}$ \\
\hline 0.8 & 1 & 504 & 0.66 & 0.85 & 400 & 6 & 9 \\
& 2 & 595 & 0.693 & 0.85 & 680 & 6 & 9 \\
0.6 & 3 & 678 & 0.74 & 0.89 & 932 & 7 & 11 \\
& 4 & 795 & 0.77 & 0.955 & 1021 & 9 & 13 \\
0.4 & 5 & 894 & 0.801 & 0.955 & 1342 & 10 & 13 \\
& 6 & 978 & 0.84 & 0.97 & 1701 & 11 & 14 \\
0.2 & 7 & 1064 & 0.89 & 0.985 & 2030 & 12 & 14 \\
\hline
\end{tabular}
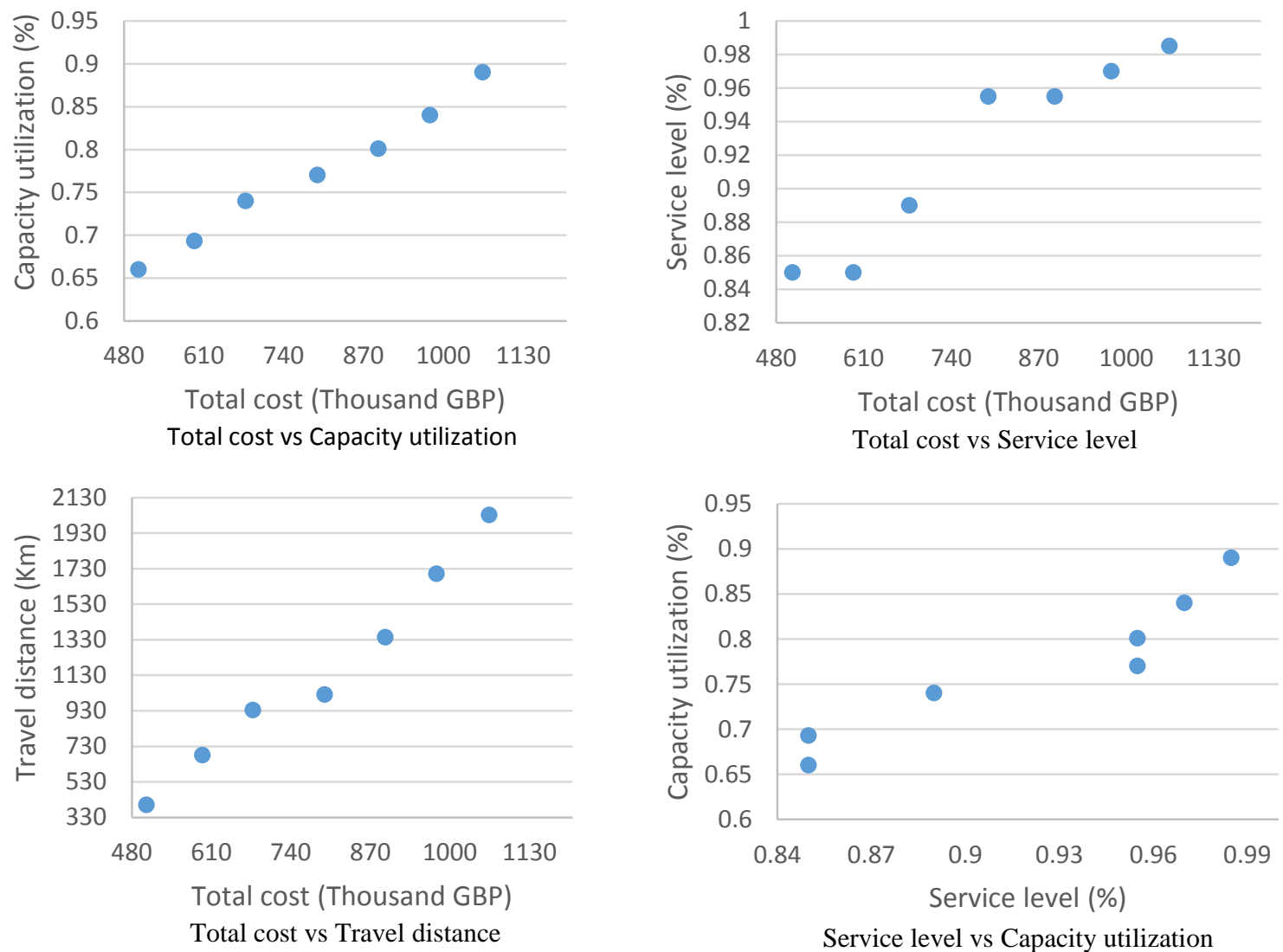

Figure 2. Comparison among the obtained objective function values. 